\title{
Article
}

\section{The Study of Maturational Timing Effect in Elite Portuguese Adolescent Basketball Players: Anthropometric, Functional and Game Performance Implications}

\author{
Isabel Fragoso ${ }^{1, *} \mathbb{D}$, Sérgio Ramos ${ }^{2}{ }^{\mathbb{D}}$, Júlia Teles ${ }^{1}{ }^{\mathbb{D}}$, Anna Volossovitch ${ }^{1}$, António Paulo Ferreira ${ }^{1}$ \\ and Luís Miguel Massuça ${ }^{1,2,3}$ \\ 1 CIPER, Faculdade de Motricidade Humana, Universidade de Lisboa, 1499-002 Cruz-Quebrada, Portugal; \\ jteles@fmh.ulisboa.pt (J.T.); anavol@fmh.ulisboa.pt (A.V.); apaulo@fmh.ulisboa.pt (A.P.F.); \\ luis.massuca@ulusofona.pt (L.M.M.) \\ 2 Faculty of Physical Education and Sport, Universidade Lusófona, 1749-024 Lisbon, Portugal; \\ sergio6ramos@hotmail.com \\ 3 ICPOL, Higher Institute of Police Sciences and Internal Security, 1749-024 Lisbon, Portugal \\ * Correspondence: ifragoso@fmh.ulisboa.pt; Tel.: +351-914019110
}

check for updates

Citation: Fragoso, I.; Ramos, S.; Teles, J.; Volossovitch, A.; Ferreira, A.P.; Massuça, L.M. The Study of Maturational Timing Effect in Elite Portuguese Adolescent Basketball Players: Anthropometric, Functional and Game Performance Implications. Appl. Sci. 2021, 11, 9894. https:// doi.org/10.3390/app11219894

Academic Editor: José Miguel Martínez-Sanz

Received: 14 July 2021

Accepted: 12 October 2021

Published: 22 October 2021

Publisher's Note: MDPI stays neutral with regard to jurisdictional claims in published maps and institutional affiliations.

Copyright: (C) 2021 by the authors Licensee MDPI, Basel, Switzerland This article is an open access article distributed under the terms and conditions of the Creative Commons Attribution (CC BY) license (https:/ / creativecommons.org/licenses/by/ $4.0 /)$.
Featured Application: The methodological classification is not important when comparing the morphological and physical capacity differences between maturity contrasting groups. Whenever possible use specific cut-off points (Preece Baines 1) to correctly classify players in contrasting maturational groups. Where game performance is concerned, and when specific population cut-off points are not available, as Lisbon boys have earlier APHV, it is recommended to use Portuguese mean APHV value or the mean average cut-off value of APHV of the sample itself, to avoid an extreme classifying bias with the suppression of late maturers.

Abstract: To estimate age at peak height velocity (APHV) in Portuguese male adolescents and; test the effect of maturational status in physical fitness attributes and individual game performance of U-14 basketball players. One mixed-longitudinal cohort with a sample of 439 Portuguese male adolescents and; one cross sectional sample of 172 Portuguese elite U-14 basketball players. The on-time group was defined within a 1-year band (more or less) from mean APHV. Preece-Baines model 1 was adopted to fit the mean stature curve. Independent samples t-tests and Kruskal-Wallis tests were performed and effect size (partial eta square) was calculated. The growth fitting revealed an APHV around 13.45 years for Portuguese male adolescents. Early maturing players exceeded significantly their peers in body size and composition, jump power and upper body strength. Early and on-time maturing players scored better in rebounds won per game (APHV = 13.81, 13.30 years), Performance Index Rating (PIR) and points scored per minute. Maturation influences morphology attributes and strength regardless of the APHV (13.81, 13.45, 13.30 years) used to obtain the cut-off points. It is recommended to use the new Portuguese mean APHV value to avoid extreme classifying bias groups.

Keywords: youth basketball; maturational status; maturity offset; Performance Index Rating; strength and conditioning

\section{Introduction}

Basketball is a dynamic and complex team sport, characterized by explosive kinetic patterns, so it is not surprising that in youth basketball the maturity status can strongly influence (a) individual physical and game performance [1], and (b) the selection process, which usually resulting in an over-representation of early maturing players in youth selection teams. Although considerable research on youth basketball players has been devoted to the relationship of maturational status with morphological attributes, fitness 
and game performance [1-7], the study of maturity and its implication on individual game performance has been, so far, inconsistent $[1,6,8,9]$.

The identification of performance-related attributes of young basketball players is a challenging task. The growth spurt, in male adolescent athletes, usually occurs between 12 and 15-years of age [8]. During this period of growth, chronological age can differ as much as 4 or 5 years from a biological age [8]. This biological difference can result in great physical fitness advantages for early maturing boys, as in the case of basketball [7,9].

Maturity is commonly assessed using methods such as skeletal age, percentage of adult height attained, or age at peak height velocity (APHV) [2,10]. Longitudinal studies are required to identify primary biological indicators of adolescent growth spurt, as age at the maximum rate of growth (9.0-15.0 years of age for girls and 11.5-17.3 years of age for boys) $[8,11]$, and magnitude of the PHV (cm/year) [12]. However, longitudinal samples are unique, limited, relatively small, time consuming, dependent of secular changes expressed since the 1960s [13] and specially not representative of the general population.

Thus, given the difficulties faced with longitudinal designs, Mirwald et al., (2002) [14] developed equations to predict years from PHV (maturity offset) in non-athletic and athletic populations, using a cross-sectional methodology, it has been cited close to a thousand times during the last two decades [15]. Predicted maturity offset and predicted APHV (APHV; APHV = chronological age-maturity offset) have been increasingly used in male young athletes [16], during the interval of the growth spurt (approximately 12-15 years of age), as a continuous variable to predict a dependent variable (i.e., physical activity) $[6,17]$ and to classify subjects into contrasting maturity groups [11], with good applicability among boys [18] who are average (on time) in maturation.

Maturity offset does provide an indication of timing, which can help researchers to understand players' development, based on the comparisons between timing contrasting categories. However, a great heterogeneity has been found among studies [2] regarding the criteria used to classify maturity groups. According to Sherar and coauthors $(2005)[3,19]$, the estimated age of peak height velocity (APHV) is compared to an international average and the biological age groups are constructed using 1-year intervals. Also, years to APHV (i.e., maturity offset) have been used to classify the subjects into maturity status categories (pre-PHV, mid-PHV and post-PHV), using 1-year intervals [4,20]. Furthermore, comparisons between biological maturity groups (e.g., early, average, and late maturers) can rely on a group average estimate APHV within \pm 0.5 years interval $[5,15,18,21]$.

Therefore, the vast majority of studies that write about early maturing players and their greater capacity in jumping, sprinting, or throwing and about the minor influence of the time of maturity on game performance used the Mirwald equations, to estimate maturity status although different classification techniques that may have influenced the obtained results.

Thus, this study was designed (a) to estimate the age at peak height velocity mean in Portuguese boys and to check if it differs from the APHV of the Mirwald study (13.81 years of age) [14] and from the predicted APHV mean of boys basketball players; and (b) to test if physical fitness attributes and individual game performance of 13-14-years-old Portuguese elite regional basketball players differ among the contrasting maturational categories obtained through different cut-off values of APHV.

\section{Materials and Methods}

\subsection{Experimental Design}

Maturity process can strongly influence the development of physical attributes of basketball players and their individual game performance. The vast majority of studies that addressed this topic used the Mirwald equations, although using different cut-off values of APHV (international, national or mean average sample) and classification time which, may or may not, influence the obtained results. Thus, this is an observational and analytical study designed to test if physical and game performance differences between maturity contrasting groups were reliant on the cut-off values of APHV selected. 
The protocol included two different studies: Study 1, with a sample of 439 boys aged between 11-16-years of age, was designed only to establish a Portuguese boys cut-off points of APHV (Figure 1). The Preece Baine 1 growth model was adjusted to the sample (study 1) mean profile. Study 2, included a sample of 172 Portuguese elite regional basketball players aged 13-14-years of age and was designed to compare the morphologic, physical and individual game performance across maturity timing categories. Anthropometric measures were measured following the International Society for the Advancement of Kinanthropometry (ISAK) protocol [22]. To avoid the influence fatigue has on players' results, morphologic and fitness measurements took place on the first day of the tournament. Before the fitness tests all subjects performed a 20-min standardized warm-up routine, and between tests the players were allowed 10-min passive rest. Each participant was verbally instructed and encouraged to give his maximum effort. All players completed seven fitness tests, from which nine variables were collected for analysis (Table 1). For more detailed description of the procedures see Ramos and coauthors (2020) [23].

\begin{tabular}{|c|c|c|c|c|c|c|c|c|c|c|c|c|c|c|c|c|}
\hline \multirow{2}{*}{$\begin{array}{l}\text { Data (source) } \\
\text { Age (years) }\end{array}$} & \multicolumn{10}{|c|}{ Auto Report (from Portuguese Health Card) } & \multicolumn{6}{|c|}{ Project FCT/MCTES(PIDDAC) ${ }^{(a)}$} \\
\hline & 1.5 & 2.5 & 3.5 & 4.5 & 5.5 & 6.5 & 7.5 & 8.5 & 9.5 & 10.5 & 11 & 12 & 13 & 14 & 15 & 16 \\
\hline $\mathrm{N}$ & 214 & 197 & 197 & 160 & 149 & 197 & 124 & 120 & 127 & 131 & \multicolumn{6}{|c|}{439} \\
\hline Cohort 1 & & & & & & & & & & $\rightarrow$ & 56 & & & & & \\
\hline Cohort 2 & & & & & & & & & & & & 87 & & & & \\
\hline Cohort 3 & & & & & & & & & & & & & 93 & & & \\
\hline Cohort 4 & & & & & & & & & & & & & & 70 & & \\
\hline Cohort 5 & & & & & & & & & & & & & & & 76 & \\
\hline Cohort 6 & - & & & & & & & & & & & & & ......... & $\ldots \ldots$ & 57 \\
\hline
\end{tabular}

Figure 1. Distribution of self-reported data (Portuguese Health Card) and performed data (cohorts). Frequency of cases by age.

Table 1. Intraclass correlation statistics for inter-rater reliability for the physical tests.

\begin{tabular}{cccc}
\hline & ICC & \multicolumn{2}{c}{$\mathbf{9 5 \%}$ CI } \\
\cline { 3 - 4 } & & Lower Bound & Upper Bound \\
\hline 20-m speed test & 0.937 & 0.921 & 0.949 \\
T-test & 0.946 & 0.929 & 0.959 \\
CMJ heigth & 0.962 & 0.951 & 0.969 \\
CMJ mean power & 0.992 & 0.990 & 0.994 \\
CMJ-S height & 0.958 & 0.947 & 0.966 \\
CMJ-S mean power & 0.980 & 0.975 & 0.984 \\
2-kg MBT test & 0.968 & 0.960 & 0.974 \\
HG rigth hand & 0.980 & 0.975 & 0.984 \\
HG left hand & 0.980 & 0.975 & 0.984 \\
Sit and reach test & 0.922 & 0.903 & 0.938 \\
\hline
\end{tabular}

${ }^{\mathrm{b}}$ ICC estimates and their 95\% confidence intervals were calculated using SPSS statistical package version 22.0 based on a consistency, two-way random-effect model. CI: Confidence interval, CMJ: Countermovement jump, CMJ-S: Countermovement jump with arms swing, ICC: Intraclass correlation, HG: handgrip, MBT: Medicine ball throw.

The participants (study 2) were assigned to three maturity timing groups based on differences between the reference APHV and their estimated APHV. We used three references for APHV: (1) the international reference used in Mirwald study, 13.81 years; (2) our national (study 1) reference, around 13.45 years, and (3) the mean value of the studied sample (study 2) with an estimate average APHV around 13.30. Independent samples t-tests and Kruskal-Wallis One-way Analysis of Variance by Ranks tests were performed to test the effect of chronological age (decimal age) and maturational status on morphological, fitness and game performance variables. 


\subsection{Subjects}

A mixed-longitudinal study (six cohorts) with a sample of 439 boys aged between 11-16, from three public schools (Oeiras, Lisboa, Loures), of Lisbon district (Portugal, Europe), based on retrospective auto report (Personal Health Record). The auto reported data included in this sample was compared and validated taking into account the crosssectional values obtained at extreme ages (11-16 years) of each subject, objectively measured by accreditated anthropometrists (Portuguese Foundation for Science and TechnologyProject number PTDC/DES/113156/2009). This sample (study 1) was only used to establish the cut-off points based on a new APHV of Portuguese boys (Figure 1).

Additionally, a cross-sectional study was conducted to examine the performance of male basketball players present at the 2016 Portuguese Youth Basketball Festival which is organized annually by the Portuguese Basketball Federation. A total of 172 male basketball players (age, $13.76 \pm 0.44$ years) were evaluated. This sample (study 2 ) gathered nine under14 (U-14) elite regional teams of the Portuguese First Division. All participants received clear guidelines on the objectives, procedures and methodology of this study. Only the players whose parents or legal guardians gave their written consent were permitted to participate in the study. The study was authorized by the Ethics Committee of Faculty of Human Kinetics-Universidade de Lisboa (No 53/2015) and by the Ethics Committee of Faculty of Physical Education and Sport-Universidade Lusófona (Phd ID, 101516444; 25-02-2016) and was performed according to the 2013 Helsinki Declaration.

\subsection{Practice Experience and Training Load}

Data related to players' practice experience (i.e., years of basketball practice) and training load (i.e., hours of practice per week) were recorded through a biosocial questionnaire. The questionnaire results are presented in Table 2.

Table 2. Descriptive statistics (mean and standard deviation) and results of independent samples $t$-tests to evaluate the effect of chronological age groups in U14 years basketball players' characteristics.

\begin{tabular}{|c|c|c|c|c|c|}
\hline \multirow[b]{2}{*}{ Variables } & \multicolumn{2}{|c|}{ CA Groups } & \multicolumn{3}{|c|}{ Effect of $C A$} \\
\hline & 13 Years $(n=34)$ & 14 Years $(n=138)$ & $t$ & $p$ & $\eta_{P}^{2}$ \\
\hline Practice experience (years) & $5.1 \pm 2.8$ & $5.4 \pm 2.5$ & -0.754 & 0.452 & 0.003 \\
\hline Training load (h/week) & $6.0 \pm 1.5$ & $6.3 \pm 1.6$ & -1.020 & 0.309 & 0.006 \\
\hline CA (years) & $13.1 \pm 0.4$ & $13.9 \pm 0.2$ & -11.561 & $<0.001$ & 0.641 \\
\hline Maturity offset (years) & $-0.37 \pm 0.78$ & $0.67 \pm 0.67$ & -7.829 & $<0.001$ & 0.265 \\
\hline APHV (years) & $13.4 \pm 0.6$ & $13.3 \pm 0.6$ & 1.226 & 0.222 & 0.009 \\
\hline \multicolumn{6}{|l|}{ MORPHOLOGY } \\
\hline Body Mass (kg) & $54.4 \pm 11.9$ & $61.0 \pm 9.4$ & -3.465 & 0.001 & 0.066 \\
\hline Stature $(\mathrm{cm})$ & $166.9 \pm 9.0$ & $174.1 \pm 8.2$ & -4.490 & $<0.001$ & 0.106 \\
\hline Arm span $(\mathrm{cm})$ & $170.0 \pm 11.7$ & $177.2 \pm 9.3$ & -3.832 & $<0.001$ & 0.080 \\
\hline Hand span (cm) & $21.4 \pm 1.8$ & $22.3 \pm 1.5$ & -3.006 & 0.003 & 0.050 \\
\hline $\operatorname{BMI}\left(\mathrm{kg} / \mathrm{m}^{2}\right)$ & $19.3 \pm 2.8$ & $20.0 \pm 2.1$ & -1.584 & 0.115 & 0.015 \\
\hline$\% \mathrm{FM}$ & $16.7 \pm 6.4$ & $16.9 \pm 5.5$ & -0.196 & 0.845 & 0.000 \\
\hline Lean mass (kg) & $44.8 \pm 7.5$ & $50.3 \pm 6.3$ & -4.403 & $<0.001$ & 0.102 \\
\hline \multicolumn{6}{|l|}{ FITNESS } \\
\hline V20-m (s) & $3.42 \pm 0.22$ & $3.33 \pm 0.23$ & 2.180 & 0.031 & 0.027 \\
\hline $\mathrm{TT}(\mathrm{s})$ & $10.6 \pm 0.7$ & $10.4 \pm 0.7$ & 1.363 & 0.175 & 0.011 \\
\hline CMJ Height $(\mathrm{cm})$ & $28.4 \pm 4.7$ & $30.3 \pm 5.1$ & -2.001 & 0.047 & 0.023 \\
\hline CMJ Power (w) & $640 \pm 152$ & $739 \pm 135$ & -3.726 & $<0.001$ & 0.075 \\
\hline CMJ-S Height $(\mathrm{cm})$ & $33.8 \pm 4.7$ & $36.1 \pm 5.7$ & -2.228 & 0.027 & 0.028 \\
\hline CMJ-S Power (w) & $698 \pm 163$ & $806 \pm 143$ & -3.856 & $<0.001$ & 0.080 \\
\hline SUM HG (kgf) & $64.4 \pm 17.4$ & $72.4 \pm 13.8$ & -2.497 & 0.016 & 0.046 \\
\hline $\operatorname{MBT}(\mathrm{m})$ & $4.49 \pm 0.71$ & $5.09 \pm 0.73$ & -4.305 & $<0.001$ & 0.098 \\
\hline Seat and reach $(\mathrm{cm})$ & $-1.8 \pm 7.6$ & $-1.1 \pm 7.9$ & -0.447 & 0.655 & 0.001 \\
\hline
\end{tabular}


Table 2. Cont.

\begin{tabular}{cccccc}
\hline & \multicolumn{2}{c}{ CA Groups } & \multicolumn{2}{c}{ Effect of CA } \\
\hline Variables & 13 Years $(\boldsymbol{n}=\mathbf{3 4})$ & 14 Years $(\boldsymbol{n}=\mathbf{1 3 8})$ & $\boldsymbol{t}$ & $\boldsymbol{p}$ & $\boldsymbol{\eta}_{\boldsymbol{P}}^{2}$ \\
\hline GAME PERFORMANCE & & & & & \\
PIR/game & $0.67 \pm 2.67$ & $2.50 \pm 3.39$ & -2.927 & 0.004 & 0.048 \\
PIR/min & $0.04 \pm 0.23$ & $0.14 \pm 0.24$ & -2.228 & 0.027 & 0.028 \\
Points/game & $2.77 \pm 1.87$ & $4.02 \pm 2.67$ & -3.178 & 0.002 & 0.038 \\
Points/min & $0.23 \pm 0.12$ & $0.26 \pm 0.16$ & -1.309 & 0.192 & 0.004 \\
Rebounds/game & $1.97 \pm 1.53$ & $2.98 \pm 1.88$ & -2.909 & $<0.001$ & 0.071 \\
Minutes played/game & $11.9 \pm 3.7$ & $14.5 \pm 3.7$ & -3.607 & $<0.07$ \\
\hline
\end{tabular}

APHV, age at peak height velocity; BMI, body mass index; CA, chronological age; CMJ, countermovement jump; CMJ-S, countermovement jump with arms swing; MBT, medicine ball throw; SUM HG, sum of right and left handgrip; PIR, Performance Index Rating; TT, T-test; V20-m, speed $20 \mathrm{~m}$ test; \%FM, fat mass percentage.

\subsection{Chronological Age and Biological Maturity}

Chronological age (CA, in decimals) was calculated and the CA group was defined considering a range midpoint, e.g., 13 years group indicate a chronological age between 12.50 and 13.49 .

Maturity offset (time before or after APHV) was predicted from a sex-specific equation [14]. Predicted APHV (years) was calculated as the difference between CA and the predicted maturity offset at observation date [14]. Three references for APHV were used: (1) the international reference used in Mirwald study, 13.81 years; (2) our Portuguese boys (see results section) reference, around 13.45 years, and (3) the mean value of the studied sample (study 2) with an estimated average APHV around 13.30 years accordingly to [18]. To categorize the basketball players in maturity groups a 1-year band was assumed. For all the references, an on-time group was defined within a band of 1 year from mean APHV (13.30, 13.45 or 13.81 years); late maturing was defined as an APHV $>14.30,14.45$, or 14.81 years; early maturing was defined as an APHV $<12.30,12.45$, or 12.81 years.

\subsection{Anthropometry and Body Composition}

Body mass $(\mathrm{kg})$, stature $(\mathrm{cm})$, sitting height $(\mathrm{cm})$, hand span $(\mathrm{cm})$ and three skinfolds $(\mathrm{mm})$ (triceps, TRI; calf, GML; and subscapular, SBS) were measured following the International Society for the Advancement of Kinanthropometry (ISAK) protocol [22]. Leg length was calculated from the difference between stature and sitting height. Arm span was measured as the perpendicular distance between the longitudinal planes of the left and right dactylion, while hand span was measured as the greater distance between the longitudinal planes of the first and fifth fingers [24].

The body composition analysis included evaluation of fat mass (FM) and fat-free mass (FFM). The relative FM (\%FM) and the FFM $(\mathrm{kg})$ were estimated from the value of skinfolds. \%FM was calculated as the arithmetic mean of the \%FM values obtained through the equations proposed by Lohman (1986) [25] and Slaughter et al., (1988) [26]. BMI was calculated by the formula BMI $=$ Body mass $/$ Stature $^{2}\left(\mathrm{~kg} / \mathrm{m}^{2}\right)$.

2.6. Physical Fitness (Speed, T-Test, Jump Tests, Medicine Ball Throw, Handgrip, Sit and Reach) and Basketball Game Performance)

\subsubsection{Physical Fitness}

Speed test: The $20 \mathrm{~m}$ speed test was performed according to the protocol described by Jakovljevic and colleagues (2012) [27]. T-test: T-test was used for the agility assessment [27]. Jump tests: The explosive and elastic leg strength were tested throughout counter movement jump (CMJ) and counter movement jumps with arm swing (CMJ-S), following the protocol described by Bosco and co-authors (1983) [28]. $2 \mathrm{~kg}$ medicine ball throw: The upper-limb explosive strength was tested using the $2 \mathrm{~kg}$ medicine ball throw (MBT). The distance (in $\mathrm{cm}$ ) attained in the best of two attempts was considered [29]. Handgrip strength: The handgrip (HG) strength was assessed with a dynamometer (Takei Physical 
Fitness Test, TKK 5001, GRIP-A) [30]. Sit and reach test: The flexibility was assessed using the sit and reach test. Each subject was seated barefoot on the floor with legs straight out ahead and with their feet placed with the soles flat against the sit and reach box. With hands on top of each other and palms facing down, each player tried to reach forward along the measuring line as far as possible. The score of the test was recorded to the nearest centimeter as the distance reached by the tip of the fingers. The vertical line of the feet soles was considered as a plane counted as $0 \mathrm{~cm}$. Negative and positive centimeters were considered when players reached forward, respectively, before and after this vertical plane [31]. The interclass correlation coefficient of all physical fitness tests are presented in Table 1.

\subsubsection{Basketball Game Performance}

Basketball individual performance was assessed in terms of minutes played per game, points scored in the tournament (i.e., expressed as the average points per game), rebounds captured in the tournament (i.e., expressed as the average rebounds per game), and by the Performance Index Rating (PIR) of each player, per game or per minute, calculated from the data recorded by the officials of the Portuguese Basketball Federation using the following formula: PIR $=$ (points + rebounds + assists + steals + blocks $)-($ missed field goals + missed free throws + turnovers + shots rejected $)$.

\subsubsection{Modelling and Statistical Analysis}

The Statistical Package for the Social Sciences software (SPSS software version 24.0, IBM SPSS, Chicago, IL, USA) was used for statistical analysis. Significance level was set at $5 \%$ with effect sizes (partial eta square, $\eta_{P}^{2}$ ) also calculated.

The Preece Baines 1 Growth Model was first considered [18,23],

$$
y=h_{1}-\frac{2)\left[\left(h_{1}-h_{\theta}\right)\right.}{\exp \left[s_{0}(t-\theta)\right]+\exp \left[s_{1}(t-\theta)\right]},
$$

where $y$ is the stature $(\mathrm{cm}), t$ is the age (years), and $h_{1}$ (adult stature), $h_{\theta}, s_{0}, s_{1}$ and $\theta$ are the mathematical parameters that were used to estimate some biological parameters (age at the peak height velocity, age at take-off, height at the peak height velocity, height at take-off, velocity at peak height velocity, velocity at take-off, increment of stature from peak height velocity to adult stature and intensity of take-off). Preece-Baines model 1 is most often used to longitudinal data, but it can be applied to cross-sectional data as presented by Zemel and Johnston (1994) [32]. These authors showed that Preece-Baines model 1 can accurately estimate the age at peak height velocity in males, as well as other characteristics, when using cross-sectional data. So, due to the cross-sectional characteristics of our data, the Preece-Baines model 1 was adjusted for the sample (study 1) mean profile.

Complementarily, basketball players descriptive' statistics (mean and standard deviation) were determined for two age groups (12.50-13.49 years and 13.50-14.49 years) and for three maturational status groups (early, on-time, late) obtained through three different cut-off points of APHV (i.e., 13.30,13.45 and 13.81 years). Independent samples t-tests and Kruskal-Wallis One-way Analysis of Variance by Ranks tests were performed to test the effect of chronological age (decimal age) and maturational status on morphological, fitness and game performance variables. Normality and homogeneity of variances were assessed using Shapiro-Wilk and Levene tests, respectively. After a significant Kruskal-Wallis test, a Dunn-Bonferroni post hoc test was performed to identify between which maturity groups the morphological, fitness and performance differences were effective. The effect size was estimated using partial eta squared $\left(\eta_{P}^{2}\right)$ and the magnitude of these results was categorized according to the Cohen (1988) benchmarks, i.e.: [0.01,0.06[ (small); [0.06,0.14[ (moderate); $\geq 0.14$ (large). 


\section{Results}

Smooth distance curve, velocity curve, and parameter estimates according to PreeceBaines model 1 are presented in Figure 2. The estimated value for male adult stature was $177.9 \mathrm{~cm}$, and the estimated age and stature at peak height velocity was 13.45 years of age and $160.5 \mathrm{~cm}$ respectively
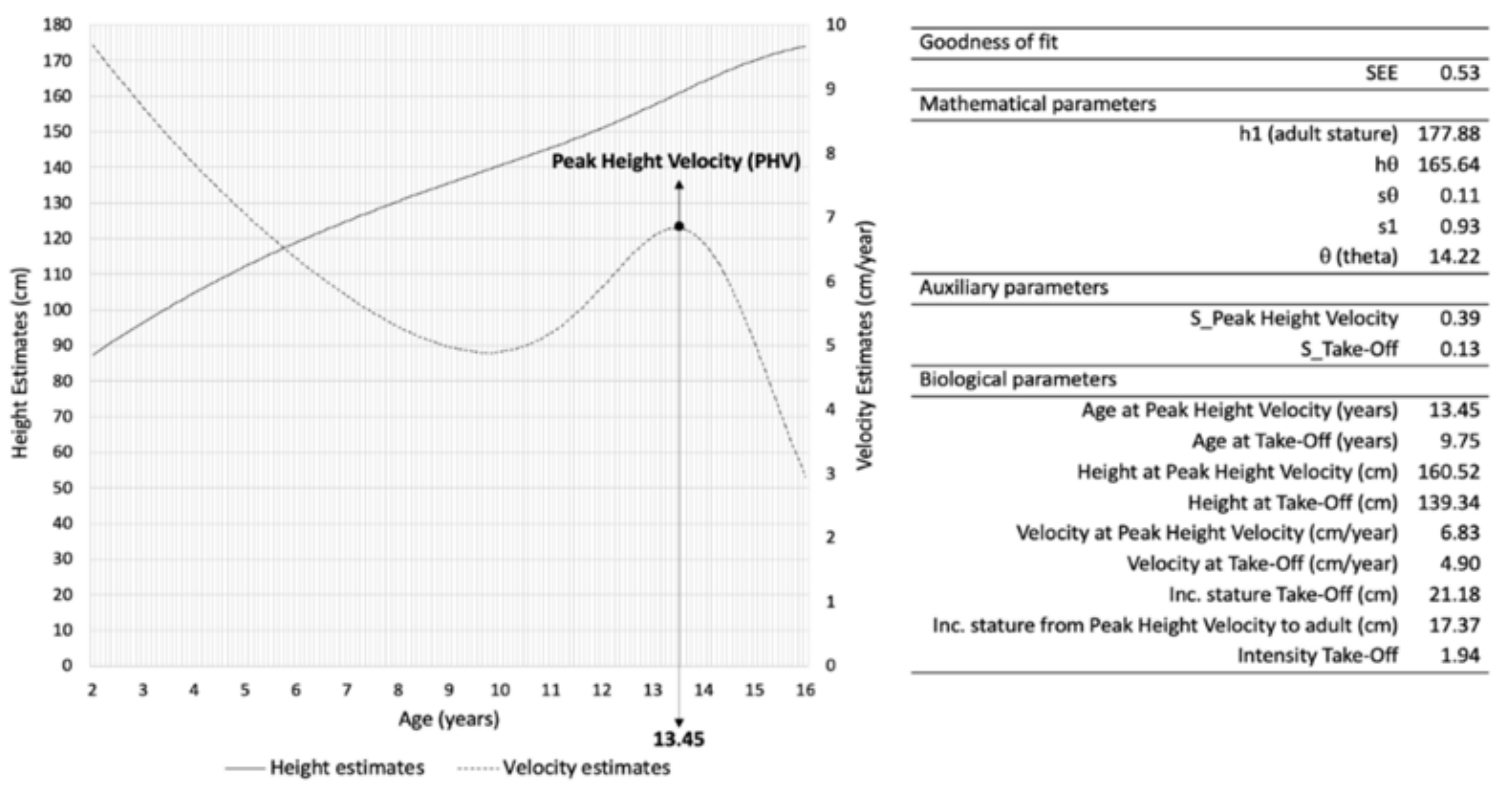

Figure 2. Smooth distance curve (height estimates), velocity curve (first derivate of the fitted function) indicating age at peak height velocity (APHV), and parameter estimates according to Preece-Baines model I.

Practice experience, training load and estimated APHV did not differ significantly between the oldest and youngest groups. Nevertheless, compared with the younger players, the older players were: (a) heavier $\left(+6.6 \mathrm{~kg}\right.$; $\left.t=-3.465, p=0.001, \eta_{P}^{2}=0.066\right)$, taller $\left(+7.2 \mathrm{~cm} ; t=-4.490, p<0.001, \eta_{P}^{2}=0.106\right)$, had more arm span $(+7.2 \mathrm{~cm} ; t=-3.832$, $\left.p<0.001, \eta_{P}^{2}=0.080\right)$, more hand span $\left(+0.9 \mathrm{~cm} ; t=-3.006, p=0.003, \eta_{P}^{2}=0.050\right)$ and more FFM $\left(+5.5 \mathrm{~kg} ; t=-4.403, p<0.001, \eta_{P}^{2}=0.102\right)$; (b) were faster $(-0.09 \mathrm{~s} ; t=2.180$, $\left.p=0.031, \eta_{P}^{2}=0.027\right)$, jumped higher on CMJ $\left(+1.9 \mathrm{~cm} ; t=-2.001, p=0.047, \eta_{P}^{2}=0.023\right)$, had more CMJ power $\left(+99 \mathrm{~W} ; t=-3.729, p<0.001, \eta_{P}^{2}=0.075\right)$, jumped higher on CMJ-S $\left(+2.3 \mathrm{~cm} ; t=-2.228, p=0.027, \eta_{P}^{2}=0.028\right)$, CMJ-S power $(+108 \mathrm{~W} ; t=-3.856, p<0.001$, $\left.\eta_{P}^{2}=0.080\right)$, and handgrip strength $\left(+8.0 \mathrm{~kg} ; t=-2.497, p=0.016, \eta_{P}^{2}=0.046\right)$, and threw the medicine ball longer $\left(+60 \mathrm{~cm} ; t=-4.305, p<0.001, \eta_{P}^{2}=0.098\right)$; and (c) had better $\mathrm{PIR} /$ game $\left(+1.83 ; t=-2.927, p=0.004, \eta_{P}^{2}=0.048\right), \mathrm{PIR} / \min (+0.10 ; t=-2.228, p=0.027$, $\left.\eta_{P}^{2}=0.028\right)$, points $/$ game $\left(+1.25 ; t=-3.178, p=0.002, \eta_{P}^{2}=0.045\right)$, rebounds/game $(+1.1$; $\left.t=-2.909, p=0.004, \eta_{P}^{2}=0.047\right)$ and play more minutes per game $(2.6 \mathrm{~min} ; t=-3.607$, $p<0.001, \eta_{P}^{2}=0.071$.

When the maturity groups were based on APHV $=13.81, \sim 22 \%$ of the subjects were early maturers, $\sim 78 \%$ were on-time, and no late mature players were found (see Table 3). When APHV = 13.45 years was used to establish maturity groups, $\sim 7 \%$ of the subjects were early maturers, and $\sim 89.5 \%$ were on-time and $\sim 3.5 \%$ were late mature. When APHV $=13.30$ years was used to establish maturity groups, $\sim 5 \%$ of the subjects were early maturers, $\sim 86 \%$ were on-time, and $\sim 9 \%$ of the subjects were early mature (see Tables 4 and 5). 
Table 3. Descriptive statistics (mean and standard deviation) and results of independent samples t-tests and Kruskal-Wallis tests to evaluate the effect of maturity groups (APHV $=13.81)$ in U14 years basketball players' characteristics.

\begin{tabular}{|c|c|c|c|c|c|}
\hline \multirow[b]{2}{*}{ Variables } & \multicolumn{2}{|c|}{ Maturity Groups (13.81) } & \multicolumn{3}{|c|}{ Effect of Maturity (13.81) } \\
\hline & Early $(n=37)$ & On-Time $(n=135)$ & $t$ & $p$ & $\eta_{P}^{2}$ \\
\hline Practice experience (years) & $4.7 \pm 2.6$ & $5.5 \pm 2.5$ & -1.79 & 0.075 & 0.019 \\
\hline Training load (h/week) & $6.7 \pm 1.8$ & $6.1 \pm 1.5$ & 2.15 & 0.033 & 0.027 \\
\hline CA (years) & $13.9 \pm 0.3$ & $13.7 \pm 0.5$ & 1.64 & 0.103 & 0.016 \\
\hline Maturity offset (years) & $1.36 \pm 0.40$ & $0.22 \pm 0.70$ & 12.75 & $<0.001$ & 0.345 \\
\hline APHV (years) & $12.5 \pm 0.2$ & $13.5 \pm 0.5$ & -17.76 & $<0.001$ & 0.451 \\
\hline \multicolumn{6}{|l|}{ MORPHOLOGY } \\
\hline Body Mass (kg) & $70.3 \pm 7.5$ & $56.8 \pm 8.9$ & 8.42 & $<0.001$ & 0.294 \\
\hline Stature $(\mathrm{cm})$ & $182.4 \pm 5.5$ & $170.0 \pm 7.6$ & 11.15 & $<0.001$ & 0.335 \\
\hline Arm span $(\mathrm{cm})$ & $185.6 \pm 6.8$ & $173.1 \pm 9.3$ & 9.11 & $<0.001$ & 0.256 \\
\hline Hand span $(\mathrm{cm})$ & $23.0 \pm 1.1$ & $21.9 \pm 1.6$ & 4.80 & $<0.001$ & 0.084 \\
\hline $\operatorname{BMI}\left(\mathrm{kg} / \mathrm{m}^{2}\right)$ & $21.1 \pm 1.9$ & $19.6 \pm 2.3$ & 3.76 & $<0.001$ & 0.077 \\
\hline$\% F M$ & $18.8 \pm 5.1$ & $16.3 \pm 5.7$ & 2.40 & 0.018 & 0.033 \\
\hline FFM (kg) & $56.8 \pm 3.9$ & $47.2 \pm 6.1$ & 11.53 & $<0.001$ & 0.324 \\
\hline \multicolumn{6}{|l|}{ FITNESS } \\
\hline V20-m (s) & $3.29 \pm 0.22$ & $3.36 \pm 0.24$ & -1.55 & 0.123 & 0.014 \\
\hline $\mathrm{TT}(\mathrm{s})$ & $10.49 \pm 0.63$ & $10.39 \pm 0.70$ & 0.82 & 0.415 & 0.004 \\
\hline CMJ Height (cm) & $30.8 \pm 4.7$ & $29.7 \pm 5.2$ & 1.15 & 0.250 & 0.008 \\
\hline CMJ Power (w) & $851 \pm 133$ & $684 \pm 125$ & 7.11 & $<0.001$ & 0.229 \\
\hline CMJ-S Height $(\mathrm{cm})$ & $36.5 \pm 4.9$ & $35.4 \pm 5.7$ & 0.99 & 0.323 & 0.006 \\
\hline CMJ-S Power (w) & $925 \pm 136$ & $747 \pm 134$ & 7.15 & $<0.001$ & 0.231 \\
\hline SUM HG (kgf) & $79.0 \pm 13.5$ & $68.5 \pm 14.4$ & 3.96 & $<0.001$ & 0.085 \\
\hline $\operatorname{MBT}(\mathrm{m})$ & $5.60 \pm 0.63$ & $4.80 \pm 0.70$ & 6.30 & $<0.001$ & 0.189 \\
\hline Seat and reach $(\mathrm{cm})$ & $-2.7 \pm 8.5$ & $-0.8 \pm 7.6$ & -1.30 & 0.195 & 0.010 \\
\hline \multicolumn{6}{|l|}{ GAME PERFORMANCE } \\
\hline PIR/game & $3.04 \pm 4.02$ & $1.90 \pm 3.10$ & 1.86 & 0.065 & 0.020 \\
\hline $\mathrm{PIR} / \mathrm{min}$ & $0.16 \pm 0.30$ & $0.11 \pm 0.22$ & 1.10 & 0.274 & 0.007 \\
\hline Points/game & $3.94 \pm 2.74$ & $3.72 \pm 2.54$ & 0.45 & 0.653 & 0.001 \\
\hline Points/min & $0.26 \pm 0.14$ & $0.26 \pm 0.15$ & -0.05 & 0.962 & 0.000 \\
\hline Rebounds/game & $3.59 \pm 2.32$ & $2.56 \pm 1.65$ & 3.04 & 0.003 & 0.052 \\
\hline Minutes played/game & $14.0 \pm 3.9$ & $14.0 \pm 3.8$ & 0.03 & 0.976 & 0.000 \\
\hline
\end{tabular}

APHV, age at peak height velocity; BMI, body mass index; CA, chronological age; CMJ, countermovement jump; CMJ-S, countermovement jump with arms swing; MBT, medicine ball throw; SUM HG, sum of right and left handgrip; PIR, Performance Index Rating; TT, T-test; V20-m, speed $20 \mathrm{~m}$ test; $\% \mathrm{FM}$, fat mass percentage; FFM, fat-free mass.

Table 4. Descriptive statistics (mean and standard deviation) and results of Kruskal-Wallis tests to evaluate the effect of maturity groups $(\mathrm{APHV}=13.45)$ in $\mathrm{U} 14$ years basketball players' characteristics.

\begin{tabular}{|c|c|c|c|c|c|c|}
\hline \multirow[b]{2}{*}{ Variables } & \multicolumn{3}{|c|}{ Maturity Groups (13.45) } & \multicolumn{3}{|c|}{ Effect of Maturity (13.45) } \\
\hline & Early $(n=12)$ & On-Time $(n=154)$ & Late $(n=6)$ & $H$ & $p$ & $\eta_{P}^{2}$ \\
\hline Practice experience (years) & $4.6 \pm 2.5$ & $5.4 \pm 2.6$ & $6.5 \pm 2.3$ & 2.42 & 0.298 & 0.014 \\
\hline Training load (h/week) & $6.9 \pm 2.0$ & $6.2 \pm 1.5$ & $5.3 \pm 0.8$ & 4.20 & 0.122 & 0.024 \\
\hline CA (years) & $13.8 \pm 0.4$ & $13.8 \pm 0.5$ & $13.8 \pm 0.5$ & 0.47 & 0.789 & 0.003 \\
\hline Maturity offset (years) & $1.6 \pm 0.4 * \#$ & $0.4 \pm 0.7 \infty$ & $-0.8 \pm 0.4$ & 36.95 & $<0.001$ & 0.179 \\
\hline APHV (years) & $12.2 \pm 0.1$ *\# & $13.3 \pm 0.5 \infty$ & $14.6 \pm 0.1$ & 48.20 & $<0.001$ & 0.222 \\
\hline \multicolumn{7}{|l|}{ MORPHOLOGY } \\
\hline Body Mass (kg) & $74.5 \pm 9.8 * \#$ & $59.3 \pm 8.8 \infty$ & $39.8 \pm 3.3$ & 34.60 & $<0.001$ & 0.170 \\
\hline Stature $(\mathrm{cm})$ & $186.3 \pm 4.6$ *\# & $172.1 \pm 8.0 \infty$ & $159.8 \pm 5.2$ & 36.97 & $<0.001$ & 0.180 \\
\hline Arm span $(\mathrm{cm})$ & $189.0 \pm 6.8 * \#$ & $175.4 \pm 9.3 \infty$ & $158.7 \pm 3.6$ & 33.34 & $<0.001$ & 0.165 \\
\hline Hand span $(\mathrm{cm})$ & $23.1 \pm 1.3 \#$ & $22.2 \pm 1.6 \infty$ & $19.9 \pm 1.3$ & 15.17 & 0.001 & 0.082 \\
\hline BMI $\left(\mathrm{kg} / \mathrm{m}^{2}\right)$ & $21.5 \pm 2.9 \#$ & $19.9 \pm 2.1 \infty$ & $15.6 \pm 1.1$ & 18.54 & $<0.001$ & 0.099 \\
\hline$\% F M$ & $20.8 \pm 6.6 \#$ & $16.8 \pm 5.4 \infty$ & $9.4 \pm 2.9$ & 16.77 & $<0.001$ & 0.090 \\
\hline FFM (kg) & $58.5 \pm 3.5 * \#$ & $49.0 \pm 6.2 \infty$ & $36.1 \pm 3.0$ & 36.60 & $<0.001$ & 0.178 \\
\hline
\end{tabular}


Table 4. Cont.

\begin{tabular}{|c|c|c|c|c|c|c|}
\hline \multirow[b]{2}{*}{ Variables } & \multicolumn{3}{|c|}{ Maturity Groups (13.45) } & \multicolumn{3}{|c|}{ Effect of Maturity (13.45) } \\
\hline & Early $(n=12)$ & On-Time $(n=154)$ & Late $(n=6)$ & $H$ & $p$ & $\eta_{P}^{2}$ \\
\hline \multicolumn{7}{|l|}{ FITNESS } \\
\hline V20-m (s) & $3.31 \pm 0.26$ & $3.34 \pm 0.23$ & $3.45 \pm 0.29$ & 1.60 & 0.450 & 0.009 \\
\hline TT (s) & $10.57 \pm 0.67$ & $10.38 \pm 0.69$ & $10.87 \pm 0.59$ & 4.45 & 0.108 & 0.026 \\
\hline CMJ Height $(\mathrm{cm})$ & $30.4 \pm 2.9$ & $30.0 \pm 5.2$ & $28.5 \pm 4.7$ & 0.60 & 0.743 & 0.004 \\
\hline CMJ Power $(w)$ & $904 \pm 93 * \#$ & $715 \pm 132 \infty$ & $477 \pm 45$ & 33.51 & $<0.001$ & 0.165 \\
\hline CMJ-S Height $(\mathrm{cm})$ & $36.2 \pm 4.2$ & $35.6 \pm 5.7$ & $34.9 \pm 5.9$ & 0.25 & 0.883 & 0.001 \\
\hline CMJ-S Power $(w)$ & $986 \pm 110 * \#$ & $779 \pm 139 \infty$ & $528 \pm 56$ & 32.44 & $<0.001$ & 0.161 \\
\hline SUM HG (kgf) & $80.4 \pm 12.3 \#$ & $70.9 \pm 14.4 \infty$ & $48.3 \pm 1.8$ & 19.00 & $<0.001$ & 0.101 \\
\hline $\operatorname{MBT}(\mathrm{m})$ & $5.49 \pm 0.77 \#$ & $4.99 \pm 0.71 \infty$ & $3.57 \pm 0.15$ & 20.13 & $<0.001$ & 0.106 \\
\hline Seat and reach $(\mathrm{cm})$ & $-4.8 \pm 7.2$ & $-1.0 \pm 7.9$ & $-0.8 \pm 5.3$ & 2.52 & 0.284 & 0.015 \\
\hline \multicolumn{7}{|l|}{ GAME PERFORMANCE } \\
\hline PIR/game & $2.46 \pm 2.44$ & $2.18 \pm 3.42$ & $0.43 \pm 2.42$ & 2.31 & 0.314 & 0.014 \\
\hline $\mathrm{PIR} / \mathrm{min}$ & $0.10 \pm 0.40$ & $0.13 \pm 0.23$ & $-0.02 \pm 0.20$ & 3.33 & 0.190 & 0.019 \\
\hline Points/game & $3.35 \pm 1.78$ & $3.84 \pm 2.66$ & $2.72 \pm 1.61$ & 1.05 & 0.593 & 0.006 \\
\hline Points/min & $0.23 \pm 0.11$ & $0.26 \pm 0.15$ & $0.18 \pm 0.09$ & 1.77 & 0.412 & 0.010 \\
\hline Rebounds/game & $3.36 \pm 1.67$ & $2.78 \pm 1.89$ & $1.70 \pm 0.85$ & 4.15 & 0.126 & 0.024 \\
\hline Minutes played/game & $13.4 \pm 3.2$ & $14.0 \pm 3.9$ & $14.2 \pm 2.8$ & 0.33 & 0.848 & 0.002 \\
\hline
\end{tabular}

APHV, age at peak height velocity; BMI, body mass index; CA, chronological age; CMJ, countermovement jump; CMJ-S, countermovement jump with arms swing; MBT, medicine ball throw; SUM HG, sum of right and left handgrip; PIR, Performance Index Rating; TT, T-test; V20-m, speed $20 \mathrm{~m}$ test; \%FM, fat mass percentage; FFM, fat-free mass. * Significant difference between early and on-time mature players, $p<0.05$. \# Significant difference between early and late mature players, $p<0.05$. $\infty$ Significant difference between on-time and late mature players, $p<0.05$.

Table 5. Descriptive statistics (mean and standard deviation) and results of Kruskal-Wallis tests to evaluate the effect of maturity group (APHV = 13.30) in U14 basketball players' characteristics.

\begin{tabular}{|c|c|c|c|c|c|c|}
\hline \multirow[b]{2}{*}{ Variables } & \multicolumn{3}{|c|}{ Maturity Groups (13.30) } & \multicolumn{3}{|c|}{ Effect of Maturity (13.30) } \\
\hline & Early $(n=8)$ & On-Time $(n=148)$ & Late $(n=16)$ & $H$ & $p$ & $\eta_{P}^{2}$ \\
\hline Practice experience (years) & $3.9 \pm 2.5 \#$ & $5.3 \pm 2.6$ & $6.7 \pm 2.2$ & 7.32 & 0.026 & 0.041 \\
\hline Training load (h/week) & $6.4 \pm 1.3$ & $6.3 \pm 1.6$ & $5.5 \pm 1.2$ & 4.71 & 0.095 & 0.027 \\
\hline CA (years) & $13.9 \pm 0.3$ & $13.8 \pm 0.5$ & $13.8 \pm 0.3$ & 0.52 & 0.772 & 0.003 \\
\hline Maturity offset (years) & $1.7 \pm 0.3 * \#$ & $0.5 \pm 0.7 \infty$ & $-0.6 \pm 0.3$ & 48.47 & $<0.001$ & 0.223 \\
\hline APHV (years) & $12.2 \pm 0.1$ *\# & $13.2 \pm 0.5 \infty$ & $14.4 \pm 0.1$ & 61.91 & $<0.001$ & 0.268 \\
\hline \multicolumn{7}{|l|}{ MORPHOLOGY } \\
\hline Body Mass (kg) & $74.3 \pm 10.6 * \#$ & $60.6 \pm 8.7 \infty$ & $43.9 \pm 4.4$ & 46.94 & $<0.001$ & 0.217 \\
\hline Stature $(\mathrm{cm})$ & $187.9 \pm 4.0 * \#$ & $173.2 \pm 7.7 \infty$ & $160.4 \pm 3.9$ & 48.34 & $<0.001$ & 0.222 \\
\hline Arm span $(\mathrm{cm})$ & $191.3 \pm 7.0 * \#$ & $176.3 \pm 9.2 \infty$ & $163.0 \pm 4.9$ & 41.28 & $<0.001$ & 0.196 \\
\hline Hand span (cm) & $22.9 \pm 1.3 \#$ & $22.3 \pm 1.6 \infty$ & $20.7 \pm 1.3$ & 17.97 & $<0.001$ & 0.096 \\
\hline BMI $\left(\mathrm{kg} / \mathrm{m}^{2}\right)$ & $21.1 \pm 3.4 \#$ & $20.1 \pm 2.1 \infty$ & $17.1 \pm 1.7$ & 25.43 & $<0.001$ & 0.131 \\
\hline \%FM & $20.2 \pm 7.8 \#$ & $17.2 \pm 5.3 \infty$ & $11.7 \pm 4.6$ & 19.44 & $<0.001$ & 0.103 \\
\hline FFM (kg) & $58.5 \pm 2.3 * \#$ & $49.9 \pm 6.1 \infty$ & $38.7 \pm 3.3$ & 47.39 & $<0.001$ & 0.219 \\
\hline \multicolumn{7}{|l|}{ FITNESS } \\
\hline V20-m (s) & $3.36 \pm 0.26$ & $3.34 \pm 0.23$ & $3.42 \pm 0.24$ & 2.60 & 0.272 & 0.015 \\
\hline $\mathrm{TT}(\mathrm{s})$ & $10.60 \pm 0.73$ & $10.38 \pm 0.69$ & $10.55 \pm 0.68$ & 1.73 & 0.421 & 0.010 \\
\hline CMJ Height (cm) & $29.5 \pm 2.5$ & $30.1 \pm 5.2$ & $28.9 \pm 5.0$ & 1.87 & 0.393 & 0.011 \\
\hline CMJ Power (w) & $887 \pm 104 * \#$ & $732 \pm 131 \infty$ & $524 \pm 72$ & 40.16 & $<0.001$ & 0.192 \\
\hline CMJ-S Height $(\mathrm{cm})$ & $35.0 \pm 4.7$ & $35.8 \pm 5.7$ & $34.4 \pm 5.2$ & 1.06 & 0.588 & 0.006 \\
\hline CMJ-S Power (w) & $963 \pm 114 * \#$ & $799 \pm 139 \infty$ & $571 \pm 67$ & 40.80 & $<0.001$ & 0.194 \\
\hline SUM HG (kgf) & $80.2 \pm 14.0 \#$ & $72.0 \pm 14.4 \infty$ & $55.0 \pm 7.8$ & 24.29 & $<0.001$ & 0.126 \\
\hline $\operatorname{MBT}(\mathrm{m})$ & $5.26 \pm 0.61 \#$ & $5.06 \pm 0.71 \infty$ & $3.99 \pm 0.54$ & 26.68 & $<0.001$ & 0.136 \\
\hline Seat and reach $(\mathrm{cm})$ & $-4.4 \pm 8.6$ & $-0.8 \pm 8.0$ & $-3.0 \pm 5.4$ & 1.98 & 0.372 & 0.012 \\
\hline
\end{tabular}


Table 5. Cont.

\begin{tabular}{|c|c|c|c|c|c|c|}
\hline \multirow[b]{2}{*}{ Variables } & \multicolumn{3}{|c|}{ Maturity Groups (13.30) } & \multicolumn{3}{|c|}{ Effect of Maturity (13.30) } \\
\hline & Early $(n=8)$ & On-Time $(n=148)$ & Late $(n=16)$ & $H$ & $p$ & $\eta_{P}^{2}$ \\
\hline \multicolumn{7}{|l|}{ GAME PERFORMANCE } \\
\hline PIR/game & $2.29 \pm 2.89$ & $2.32 \pm 3.42$ & $0.41 \pm 2.22$ & 5.29 & 0.071 & 0.030 \\
\hline $\mathrm{PIR} / \mathrm{min}$ & $0.04 \pm 0.48$ & $0.14 \pm 0.23 \infty$ & $-0.01 \pm 0.18$ & 6.62 & 0.036 & 0.038 \\
\hline Points/game & $3.01 \pm 1.74$ & $3.95 \pm 2.67$ & $2.51 \pm 1.52$ & 5.53 & 0.063 & 0.032 \\
\hline Points/min & $0.22 \pm 0.12$ & $0.27 \pm 0.15 \infty$ & $0.17 \pm 0.08$ & 7.41 & 0.025 & 0.042 \\
\hline Rebounds/game & $3.18 \pm 1.76$ & $2.89 \pm 1.90 \infty$ & $1.60 \pm 0.89$ & 9.98 & 0.007 & 0.056 \\
\hline Minutes played/game & $12.8 \pm 2.7$ & $14.1 \pm 3.9$ & $13.2 \pm 3.2$ & 1.81 & 0.405 & 0.011 \\
\hline
\end{tabular}

APHV, age at peak height velocity; BMI, body mass index; CA, chronological age; CMJ, countermovement jump; CMJ-S, countermovement jump with arms swing; MBT, medicine ball throw; SUM HG, sum of right and left handgrip; PIR, Performance Index Rating; TT, T-test; V20-m, speed $20 \mathrm{~m}$ test; \%FM, fat mass percentage; FFM, fat-free mass. * Significant difference between early and on-time mature players, $p<0.05$. \# Significant difference between early and late mature players, $p<0.05$. $\infty$ Significant difference between on-time and late mature players, $p<0.05$.

Practice experience and training volume did not differ between maturational status groups, when considering APHV $=13.30,13.45$ or 13.81 years.

Results were similar for APHV $=13.30,13.45$ and 13.81 years, i.e., compared to their on-time peers, early mature players were: (a) taller $(13.30$ years, $+14.7 \mathrm{~cm}$; 13.45 years, $+14.2 \mathrm{~cm}$; 13.81 years, $+12.4 \mathrm{~cm}$ ), heavier $(13.30$ years, $+13.7 \mathrm{~kg}$; 13.45 years, $+15.2 \mathrm{~kg}$; 13.81 years, $+13.5 \mathrm{~kg}$ ), had bigger arm span (13.30 years, $+15 \mathrm{~cm}$; 13.45 years, $+13.6 \mathrm{~cm}$; 13.81 years, $+12.5 \mathrm{~cm})$, wider hand span $(13.30$ years, $+0.6 \mathrm{~cm}$; 13.45 years, $+0.9 \mathrm{~cm}$; 13.81 years, $+1.1 \mathrm{~cm}$ ), superior BMI (13.30 years, $+1.0 \mathrm{~kg} / \mathrm{m}^{2} ; 13.45$ years and 13.81 years, $+1.5 \mathrm{~kg} / \mathrm{m}^{2}$ ), presented more relative fat mass (13.30 years, $+3 \%$; 13.45 years, $+4 \%$; 13.81 years, $+2.5 \%$ ) and more FFM (13.30 years, $+8.6 \mathrm{~kg}$; 13.45 years, $+9.5 \mathrm{~kg}$; 13.81 years, $+9.6 \mathrm{~kg}$ ); (b) were better in CMJ power (13.30 years, $+155 \mathrm{~W} ; 13.45$ years, $+189 \mathrm{~W} ; 13.81$ years, $+167 \mathrm{~W}$ ), CMJ-S power (13.30 years, $+164 \mathrm{~W} ; 13.45$ years, $+207 \mathrm{~W} ; 13.81$ years, $+178 \mathrm{~W})$, handgrip strength (13.30 years, $+8.2 \mathrm{~kg}$; 13.45 years, $+9.5 \mathrm{~kg}$; 13.81 years, $+10.5 \mathrm{~kg}$ ) and threw the medicine ball further (13.30 years, $+0.2 \mathrm{~m}$; 13.45 years, $+0.50 \mathrm{~m} ; 13.81$ years, $+0.8 \mathrm{~m}$ ); and (c) had more 1.3 rebounds per game (13.81 years).

In addition, when APHV $=13.30$ was used to establish maturity groups, the players that matured on-time had significant higher scores in PIR/min, points $/ \mathrm{min}$ and rebounds/game then the players that matured late.

Tables 2-4 present the morphological attributes, fitness performance measures and specific performance variables for each maturational status group obtained when APHV was equal to $13.81,13.45$ and 13.30 years of age, respectively.

\section{Discussion}

The study of physical growth of Lisbon district boys (Portuguese) revealed an age at peak height velocity around 13.45 years of age. In addition, the study of maturational statusrelated variation of 13-14 years of age (U-14) Portuguese male elite regional basketball players, in relation to physical fitness attributes and individual game performance showed that: (a) older players and early maturing players exceed significantly their peers in body size, body composition, jump power and upper body strength (handgrip and medicine ball throw) in all sorts of maturity classification (i.e., when using 13.30, 13.45 and 13.81 as APHV); (b) among the 172 athletes (sample 2), only $5 \%, 7 \%$ and $22 \%$ of the subjects were early maturing players, and 16 players and six players $(9 \%, 3.5 \%, 0 \%)$ were late maturers when basketball player's predicted mean, Portuguese and international reference for APHV were used (i.e., 13.30, 13.45, 13,81 years); (c) older players surpassed their younger peers in PIR (per game and per minute), points scored per game and minutes played per game while early maturers performed better only in rebounds (APHV $=13.81$ years) and on-time mature players had higher values of PIR/min and points/min compared to the other maturity groups (APHV $=13.30$ years). 
Although the variety of analytical procedures; the secular growth trend, as most of longitudinal studies were done between 70 and 90; the different proportions of populations; the little variation (more or less a year) of sex-specific estimates of timing and tempo; the APHV have been frequently considered to categorize athletes according to maturity $[11,33]$. Portuguese boys' APHV, estimated with PB model 1, was 13.45 years of age with a velocity in the PHV of $6.8 \mathrm{~cm} /$ year. The mean APHV for males in this study was somewhat early compared to most of (13.8-14.3 years of age) other PB model 1 analysis [34-37]. The mean APHV for boys in the present study was also early compared to that for boys in the Wroclaw Growth Study, $14.0 \pm 1.2$ years [38]. The results suggest that our athletes might grow and develop slightly early compared to the majority of samples studied longitudinally although later than a Japan sample [33]. In contrast, the mean velocity $(6,8 \mathrm{~cm} /$ year) in the PHV was smaller when considering the aforementioned samples (range 8.8-9.7 cm/year) [33-38].

This study indicates that the boy's growth spurt in stature occurs about half-year earlier in the Lisbon district (Portuguese) boys as compared to Mirwald study cut-off value (i.e., 13.81 years of age) and other European studies $[2,8]$ but later than the predicted mean APHV (13.30 years) obtain, with Mirwald equation, for our sample. These facts may suggest that the selection process of male U-14 Portuguese elite regional basketball teams, which participated in the tournament, was influenced by biological maturation. The same trend was described for the boy's categories of Spanish basketball, i.e., Spanish elite players (selected from the best and more representative teams) had a higher biological age (measured by years from APHV, i.e., maturity offset) than non-elite basketball players [7].

Maturity-related differences among male athletes are well documented in the available literature [8]. Body size dimensions have been considered important attributes for young male basketball players performance [7]. In the present study, athletes with different maturational status showed significant differences in body mass, stature, arm span and hand span with large effects sizes been observed for body mass, stature and arm span.

Moreover, early maturation is also associated with better performances $[1,8]$. In this study, early maturing basketball players performed significantly better on upper (HG and MBT) and lower (CMJ and CMJ-S power) body strength tests (effect sizes showed large effects for $\mathrm{HG}, \mathrm{MBT}, \mathrm{CMJ}$ and CMJ-S power).

Our results confirmed that chronological age and biological maturation have an impact on body size attributes and strength performances. The three cut-off points presented the same differences among groups showing that morphologic and fitness results were not dependent from the cut-off point used. Although the lack of late maturers when using 13.81 years of age cut-off point and the number of late maturers (9\%) when groups were classified using 13.30 years cut-off value, the morphologic and fitness results were similar to those previously reported, showing that maturation status explained a significant portion of body size variance and had a significant effect on jumps and upper body strength [7].

The present study found that maturational status had a significant and positive effect on morphological attributes and fitness performance, but a small influence on Performance Index Rating (PIR) and average scored points. These two scores of basketball individual performance (i.e., PIR and point average) are the game performance indicators most commonly used to evaluate player's individual performance in a basketball game $[1,7]$. However, the cut-off value 13.30 years of age (average APHV of the studied basketball players), which included a very small number of athletes (5\%) in the advanced group, showed significant differences between the on time and the late maturers, but not with the early maturity status, with only one exception, the rebounds. Thus, although some morphological and fitness attributes regarding speed and power are important attributes for basketball success, our results suggested, in accordance with Ostojic et al., (2006) [9], that these attributes, by themselves, are not the drive for excellence in basketball.

Torres-Unda and coauthors (2016) [1] observed: (a) that stature and maturity offset were the best predictors of individual performance in basketball; and (b) a moderate correlation between maturity offset and PIR per game. In the present study, maturity status groups showed no effect on player individual performance (evaluated by PIR) and points 
scored (per game and per minute), but was not used as a predictor nor as a covariate. In addition, maturity status (only for maturity groups based on 13.30 and 13.81 years of age cut-off points) had a significant effect on average rebound. Rebounding involves previous physical contact, and for that very reason, it is expected that heavier and taller players, with greater jump power would be able to win more rebounds per game. This observation reinforces that, in the complex dynamic of a basketball game it is highly possible that other factors (e.g., technique, tactics, strategy or psychological features) play a key influence on individual player's performance [9]. Also, performance may be independent from biological maturation [8] or may not be directly related with most of the performance variables. Instead, it may possibly moderate the impact of other factors (like years of experience, chronological age, strategy or psychological features) on performance.

This study showed that the classification technique chosen to obtain the maturational groups is an important issue. The methodologies usually referred to obtain the different maturity groups proceed from an international reference such as Sherar et al., (2005) [19] or from the mean average cut-off value of APHV of the sample itself, as Wickel et al., (2009) [18] recommended (regardless of the time frame of 6 months or a year). These classification methodologies did not change the morphological and physical fitness differences obtained between maturational groups. However, the differences obtained for game performance only happened when the maturity-categories bias was larger and not always between the extreme groups, showing that the classification methodology interfered on the variability of results.

Accordingly, it seems advisable to use whenever possible references obtained from a specific longitudinal sample. If this is not the case then one can choose the average cut-off value of APHV of the sample itself, accordingly to Wickel et al., (2009) [18], to avoid an extreme classifying bias with the suppression of late maturers. In case we want to compare several unique samples (in sporting and morphological terms) and when there is a need for an independent reference, it is recommend to use a specific reference, since populations have specific growth characteristics not always in accordance with the reference cut-off points of APHV used by Sherar et al., (2005) [19] as shown when Portuguese PHV was compared with other European populations [2,8].

It might also be worth mentioning, though, that one of the limitations of this study is the analysis of two distinct cohorts. Data from study 1 was obtained from three public schools and data from study 2 from 9 regional U14 teams of elite basketball athletes. Although we performed a cross-sectional validation, as we used the height values included in the Personal Health Report, the clinical value of the PB1 model is dependent on the errors made during the assessment of the child's height measurements. Obviously, the influence of measurement errors will be greater when fewer measurements are performed on each child, which prevented us from using children with less than 11 successive assessments. Therefore, Preece-Baines model 1 was adjusted to the average profile of 439 boys aged between 11-16 and not to an average profile of elite male basketball players.

Longitudinal samples are unique and may not be representative of specific populations, thus a Preece-Baines model 1 should further be fitted to a more specific population, as we have already mentioned above. Data from Study 1 and Study 2 dated from 2012 and 2016, respectively, however the longitudinal samples used to develop the Mirwald equation dated from the 1960s to the 1990s. Secular changes in height have occurred in European populations since the 1960s [10,13] however this phenomenon have declined or stopped in many countries.

All predictions have associated errors, especially if the selected samples are from adolescent athletes. Inter-individual differences in the timing and tempo of growth spurt are considerable and the advanced maturity status of male athletes can limit the use of predicted APHV $[11,33,39]$. The protocol for the prediction equation was based on Europeans samples (Caucasians, Whites) however, basketball players, on average, have proportionally longer lower limbs [7]. This population variation in the proportions of sitting height and lower limbs length implies that we must be careful when applying the 
Mirwald protocol [8]. Although the average predicted APHV for the sample in question (13.30 years) should not be ignored, bearing in mind that the average values usually do not differ much from the average values obtained from a longitudinal sample, it would be important to collect longitudinal information on Portuguese male basketball players. It would also be of great interest to evaluate the effect of maturation on morpho-functional variables and performance (PIR) using other maturity variables like bone age or percentage of predicted adult height. Bone age and relative height are less influenced by subjects ${ }^{\prime}$ dimensions, proportions and by secular changes. Finally, maturity needs to be assigned as a moderator between physical and fitness attributes and individual performance in basketball (Performance Index Rating and average scored points).

\section{Conclusions}

Our findings suggest that: (a) the APHV of boys of Lisbon population was halfyear earlier as compared to Mirwald study cut-off value (i.e., 13.81 years) and other European studies; (b) the APHV (maturation) influences morphological attributes and fitness (strength) performance, regardless of the cut-off points used to classify the sample units in contrasting maturity groups, with early maturing players exceeding significantly their peers in body size, body composition and strength performances; and (c) maturity status does not seem to influence individual game performance (only the rebounds seem directly related with maturation), however it is still important to continue studying this topic (d) Where game performance is concerned and when population cut-off points of APHV are not available, it is recommended to categorize the sample according the Wickel and coauthors (2009) [18] classification methodology to avoid an extreme classification bias with the suppression of late maturers.

Author Contributions: Conceptualization, L.M.M. and I.F.; methodology, S.R., A.V., A.P.F., I.F. and L.M.M.; formal analysis S.R., J.T., I.F. and L.M.M.; investigation, I.F., S.R., J.T., A.V., A.P.F. and L.M.M.; Resources, A.P.F., S.R., I.F.; writing—original draft preparation, I.F. and S.R.; writing—review and editing, I.F., S.R., A.V., A.P.F. and L.M.M. All authors have read and agreed to the published version of the manuscript.

Funding: This research was funded by Fundação para a Ciência e Tecnologia (Grant number: PTDC/DES/113156/2009).

Institutional Review Board Statement: The study was conducted according to the guidelines of the Declaration of Helsinki, and approved by the Ethics Committee of the Faculty of Physical Education and Sport-Universidade Lusófona.

Informed Consent Statement: Written informed consent was obtained from all subjects involved in the study.

Data Availability Statement: Not applicable.

Acknowledgments: The authors thank Ana Lúcia Silva, Lara Costa e Silva, João Albuquerque and Carlos Barrigas from Faculty of Human Kinetics, University of Lisbon their tireless support in data collection. Finally, we would like thank all those who participated in this study namely, trainees, schoolchildren, guardians, professors, and others, such as school assistants.

Conflicts of Interest: The authors declare no conflict of interest.

\section{References}

1. Torres-Unda, J.; Zarrazquin, I.; Gravina, L.; Zubero, J.; Seco, J.; Gil, S.M.; Gil, J.; Irazusta, J. Basketball performance is related to maturity and relative age in elite adolescent players. J. Strength Cond. Res. 2016, 30, 1325-1332. [CrossRef] [PubMed]

2. Albaladejo-Saura, M.; Vaquero-Cristóbal, R.; González-Gálvez, N.; Esparza-Ros, F. Relationship between biological maturation, physical fitness, and kinanthropometric variables of young athletes: A systematic review and meta-analysis. Int. J. Environ. Res. Public Health 2021, 18, 328. [CrossRef] [PubMed]

3. Arede, J.; Fernandes, J.; Moran, J.; Norris, J.; Leite, N. Maturity timing and performance in a youth national basketball team: Do early-maturing players dominate? Int. J. Sports Sci. Coach. 2020, 16, 722-730. [CrossRef]

4. Arede, J.; Oliveira, I.; Ángel Gomez, M.A.; Leite, N. A multi-block multivariate analysis to explore the influence of the somatic maturation in youth basketball. Front. Psychol. 2021, 12, 602576. [CrossRef] [PubMed] 
5. Sekine, Y.; Hoshikawa, S.; Hirose, N. Longitudinal age-related morphological and physiological changes in adolescent male basketball players. J. Sports Sci. Med. 2019, 18, 751-757.

6. te Wierike, S.C.; Elferink-Gemser, M.T.; Tromp, E.J.; Vaeyens, R.; Visscher, C. Role of maturity timing in selection procedures and in the specialisation of playing positions in youth basketball. J. Sports Sci. 2015, 33, 337-345. [CrossRef]

7. Torres-Unda, J.; Zarrazquin, I.; Gil, J.; Ruiz, F.; Irazusta, A.; Kortajarena, M.; Seco, J.; Irazusta, J. Anthropometric, physiological and maturational characteristics in selected elite and non-elite male adolescent basketball players. J. Sports Sci. 2013, 31, 196-203. [CrossRef]

8. Malina, R.M.; Bouchard, C.; Bar-Or, O. Growth, Maturation and Physical Activity, 2nd ed.; Human Kinetics: Champaign, IL, USA, 2004

9. Ostojic, S.; Mazic, S.; Dikic, N. Profiling in basketball: Physical and physiological characteristics of elite players. J. Strength Cond. Res. 2006, 20, 640-744. [CrossRef]

10. Cameron, N.; Bogin, B. Human Growth and Development, 2nd ed.; Elsevier: London, UK, 2012.

11. Malina, R.M.; Kozieł, S.M. Validation of maturity offset in a longitudinal sample of Polish girls. J. Sports Sci. 2014, 32, 1374-1382. [CrossRef]

12. Tanner, J.; Healy, M.; Goldstein, H.; Cameron, C. Assessment of Skeletal Maturity and Prediction of Adult Height (TW3 Method); W.B. Saunders: London, UK, 2001.

13. Bodzsàr, E.B.; Susanne, C. Secular growth changes in Europe: Do we observe similar trends? Considerations for future research. In Secular Growth Changes in Europe, Bodzsàr, E.B., Susanne, C., Eds.; Eötvös University Press: Budapest, Hungary, 1998 ; pp. 369-381.

14. Mirwald, R.L.; Baxter-Jones, A.D.; Bailey, D.A.; Beunen, G.P. An assessment of maturity from anthropometric measurements. Med. Sci. Sports Exerc. 2002, 34, 689-694.

15. Guimarães, E.; Ramos, A.; Janeira, M.A.; Baxter-Jones, A.D.G.; Maia, J. How does biological maturation and training experience impact the physical and technical performance of 11-14-year-old male basketball players? Sports 2019, 7, 243. [CrossRef] [PubMed]

16. Till, K.; Cobley, S.; Wattie, N.; O’Hara, J.; Cooke, C.; Chapman, C. The prevalence, influential factors and mechanisms of relative age effects in UK Rugby League. Scand. J. Med. Sci. Sports 2010, 20, 320-329. [CrossRef]

17. Guimarães, E.; Baxter-Jones, A.; Maia, J.; Fonseca, P.; Santos, A.; Santos, E.; Tavares, F.; Janeira, M.A. The roles of growth, maturation, physical fitness, and technical skills on selection for a Portuguese under-14 years basketball team. Sports $2019,7,61$. [CrossRef]

18. Wickel, E.E.; Eisenmann, J.C.; Welk, G.J. Maturity-related variation in moderate-to-vigorous physical activity among 9-14-year olds. J. Phys. Act. Health 2009, 6, 597-605. [CrossRef] [PubMed]

19. Sherar, L.B.; Mirwald, R.L.; Baxter-Jones, A.D.G.; Thomis, M. Prediction of adult height using maturity-based cumulative height velocity curves. J. Pediatr. 2005, 147, 508-514. [CrossRef] [PubMed]

20. Carvalho, H.M.; Gonçalves, C.E.; Collins, D.; Paes, R.R. Growth, functional capacities and motivation for achievement and competitiveness in youth basketball: An interdisciplinary approach. J. Sports Sci. 2018, 36, 742-748. [CrossRef]

21. Lätt, E.; Mäestu, J.; Rääsk, T.; Purge, P.; Jürimäe, T.; Jürimäe, J. Maturity-related differences in moderate, vigorous, and moderateto-vigorous physical activity in 10-14-year-old boys. Percept. Mot. Skills 2015, 120, 659-670. [CrossRef]

22. Marfell-Jones, M.; Olds, T.; Stewart, A.; Carter, J. International Standards for Anthropometric Assessment (Revised 2006); International Society for the Advancement of Kinanthropometry: Potechefstroom, South Africa, 2006.

23. Ramos, S.; Volossovitch, A.; Ferreira, A.P.; Barrigas, C.; Fragoso, I.; Massuça, L. Differences in maturity, morphological, and fitness attributes between the better- and lower-ranked male and female U-14 Portuguese elite regional basketball teams. J. Strength Cond. Res. 2020, 34, 878-887. [CrossRef]

24. Massuça, L.; Fragoso, I. A multidisciplinary approach of success in team-handball. Apunts Med. Esport 2013, 48, 143-151. [CrossRef]

25. Lohman, T.G. Applicability of body composition techniques and constants for children and youths. Exerc. Sport Sci. Rev. 1986, 14, 325-357. [CrossRef]

26. Slaughter, M.H.; Lohman, T.G.; Boileau, R.A.; Horswill, C.A.; Stillman, R.J.; Van Loan, M.D.; Bemben, D.A. Skinfold equations for estimations of body fatness in children and youth. Hum. Biol. 1988, 60, 709-723.

27. Jakovljevic, S.; Karalejic, M.; Pajic, Z.; Macura, M.; Erculz, F. Speed and Agility of 12 and 14-year-old elite male basketball players. J. Strength Cond. Res. 2012, 26, 2453-2459. [CrossRef]

28. Bosco, C.; Luhtanen, P.; Komi, P. A simple method for measurement of mechanical power in jumping. Eur. J. Appl. Physiol. 1983, 50, 273-282. [CrossRef]

29. Delextrat, A.; Cohen, D. Strength, power speed and agility of women basketball players according to playing position. J. Strength Cond. Res. 2009, 23, 1974-1981. [CrossRef] [PubMed]

30. España-Romero, V.; Ortega, F.B.; Vicente-Rodríguez, G.; Artero, E.G.; Rey, J.P.; Ruiz, J.R. Elbow position affects handgrip strength in adolescents: Validity and reliability of Jamar, DynEx, and TKK dynamometers. J. Strength Cond. Res. 2010, 24, $272-277$. [CrossRef]

31. Mayorga-Vega, D.; Merino-Marban, R.; Viciana, J. Criterion-related validity of sit-and-reach tests for estimating hamstring and lumbar extensibility: A meta-analysis. J. Sports Sci. Med. 2014, 13, 1-14. 
32. Zemel, B.S.; Johnston, F.E. Application of the Preece-Baines growth model to cross-sectional data: Problems of validity and interpretation. Am. J. Hum. Biol. 1994, 6, 563-570. [CrossRef]

33. Malina, R.M.; Coelho-e-Silva, M.J.; Figueiredo, A.J.; Philippaerts, R.M.; Hirose, N.; Peña Reyes, M.E.; Gilli, G.; Benso, A.; Vaeyens, R.; Deprez, D.; et al. Tanner-Whitehouse Skeletal Ages in Male Youth Soccer Players: TW2 or TW3? Sports Med. 2018, 48, 991-1008. [CrossRef] [PubMed]

34. Kimura, J.; Tachibana, K.; Imaizumi, K.; Kurosawa, K.; Kuroki, Y. Longitudinal growth and height velocity of Japanese children with Down's syndrome. Acta Paediatr. 2003, 92, 1039-1042. [CrossRef]

35. Largo, R.; Prader, A. Pubertal development in Swiss girls. Helv. Paediatr. Acta 1983, 38, 229-243. [PubMed]

36. Lee, P.A. Normal ages of pubertal events among American males and females. J. Adolesc. Health Care 1980, 1, 26-29. [CrossRef]

37. Tanner, J.; Whitehouse, R. Clinical longitudinal standards for height, weigth, height velocity, weight velocity and stages of puberty. Arch. Dis. Child. 1976, 51, 170-179. [CrossRef] [PubMed]

38. Malina, R.M.; Bielicki, T. Growth and maturation of boys active in sports: Longitudinal observations from the Wroclaw Growth Study. Pediatr. Exerc. Sci. 1992, 4, 68-77. [CrossRef]

39. Malina, R.M.; Kozieł, S.M. Validation of maturity offset in a longitudinal sample of Polish boys. J. Sports Sci. $2014,32,424-437$. [CrossRef] [PubMed] 